\title{
Factores asociados a la malignización del liquen plano oral. Revisión de la literatura
}

\section{Factors associated with the malignization of oral lichen planus. Review of the literature.}

\author{
E. Asensi Anta*, S. Sardañés Martínez*, K. Izquierdo Gómez*, \\ E. Jané Salas**, A. Estrugo Debesa**, J. López López******.
}

\section{RESUMEN}

Introcucción: El liquen plano (LP) es una enfermedad sistémica autoinmune de etiología desconocida y curso crónico que puede resultar en el desarrollo de carcinoma oral de células escamosas (COCE).

Objetivo: Evaluar la evidencia relacionada con la malignización del LPO, así como las características clínicas y la incidencia de las lesiones que pueden desarrollan un COCE.

Material y métodos: Se revisa la base de datos MEDLINE PubMed de los últimos 5 años (2013-2018) utilizando las siguientes palabras clave: "malignant transformation of oral lichen planus" and "squamous cell oral carcinoma".

Resultados: Se obtuvieron 48 artículos de los cuales tras la lectura resúmenes y la aplicación de nuestros criterios de inclusión y exclusión obtuvimos un total de 19 artículos, además de los 4 artículos añadidos mediante búsqueda manual. La revisión se basa en la comparación de 9 estudios de cohortes para analizar la incidencia de la progresión de LPO a COCE.

Conclusiones: Debemos considerar el LPO como una enfermedad potencialmente maligna (0.5- $1 \%$ ) siendo el sexo femenino, el tipo erosivo y la localización en la lengua factores de riesgo para la transformación a COCE. Teniendo en cuenta el riesgo de malignización es esencial el control de las lesiones para poder realizar un diagnóstico precoz.

PALABRAS CLAVE: "transformación maligna del liquen plano"; "carcinoma de células escamosas"; "liquen plano oral"; "cáncer oral".

\begin{abstract}
Introduction: Lichen planus (LP) is a systemic autoimmune disease of unknown etiology and chronic course that can result in the development of oral squamous cell carcinoma (OECD).

Objective: To evaluate the evidence related to the malignization of the LPO as well as the clinical characteristics and the incidence of the lesions that can develop a COCE.

Material and methods: The MEDLINE PubMed database of the last 5 years (2013-2018) was reviewed using

* $\quad$ Máster en Medicina, Cirugía e Implantología Oral. Facultad de Medicina y Ciencias de la Salud (Odontología), Universidad de Barcelona.

** Profesor de Medicina Oral, Departamento de Odontoesotmoatología. Facultad de Medicina y Ciencias de la Salud (Odontología), Universidad de Barcelona. // Oral Health and Masticatory System Group, Institut d'In vestigació Biomédica de Bellvitge (IDIBELL, Bellvitge Institute of Biomedical Research), L'Hospitalet de Llo bregat, Barcelona, España.

*** Director Facultativo y Responsable del Área Médico Quirúrgica del Hospital Odontológico Universidad de Bar celona (HOUB). L'Hospitalet de Llobregat, Barcelona, España.
\end{abstract}


the following keywords: "malignant transformation of oral lichen planus" and "squamous cell oral carcinoma". Results: 48 articles were obtained, after reading the summaries and applying our inclusion and exclusion criteria, we obtained a total of 19 articles, in addition to the 4 articles added by manual search. The review is based on the comparison of 9 cohort studies to analyze the incidence of progression from LPO to COCE.

Conclusions: We should consider the LPO as a potentially malignant disease (0.5-1\%), being the female sex, the erosive type and the localization in the tongue as a risk factors for the transformation to COCE. Taking into account the risk of malignancy, it is essential to control the lesions in order to make an early diagnosis.

KEY WORDS: "malignant transformation of oral lichen planus"; "squamous cell oral carcinoma"; "Oral lichen planus"; "Oral cancer".

Fecha de recepción: 24 de mazo de 2019

Fecha de aceptación: 10 de junio de 2019

E. Asensi Anta, S. Sardañés Martínez, K. Izquierdo Gómez, E. Jané Salas, A. Estrugo Debesa, J. López López.

Factores asociados a la malignización del liquen plano oral. Revisión de la literatura. 2019; 35, (3): 131-137.

\section{INTRODUCCIÓN}

El liquen plano (LP) es una enfermedad inflamatoria mucocutánea crónica de afectación sistémica y etiología desconocida que puede afectar a la piel, el pelo, las uñas y los revestimientos mucosos. Se estima que su prevalencia oscila alrededor del 5\% y presenta mayor predilección por el sexo femenino en una proporción $2: 1^{(1)}$. Pese a que las manifestaciones clínicas del LP presentan numerosas variantes, la piel y la cavidad oral son las superficies más afectadas. El liquen plano oral (LPO) afecta al 1-4\% de la población y con mayor frecuencia a las mujeres mayores de 40 años en la quinta década de la vida ${ }^{(2)}$. Se caracteriza por episodios de reactivación y remisión (cursa a brotes) y clásicamente se presenta con lesiones reticulares bilaterales que no se desprenden al raspado ${ }^{(3)}$. El diagnóstico se basa en criterios tanto clínicos como histopatológicos. Van der Meij et al. ${ }^{(4)}$ propusieron unos criterios diagnósticos basados en la definición de la World Health Organization (WHO) (Tabla 1). De acuerdo con éstos, la presentación clínica del LPO incluye tanto lesiones de patrón reticular como sobreelevadas, erosivas, atróficas, bullosas y en placa ${ }^{(4)}$. En ocasiones pueden aparecer lesiones similares al LPO en pacientes que toman determinados fármacos como antidiabéticos, antihipertensivos y antiinflamatorios, así como en los que presentan restauraciones dentales del tipo amalgama, hábitos tóxicos o experimentan reacciones de injerto contra huésped denominándose reacciones liquenoides $(\mathrm{RL})^{(3)}$.

Clásicamente deben cumplirse tanto los criterios clínicos como los histopatológicos para diagnosticar LPO. Asimismo, en caso de no se cumplan todos las denominaremos $\mathrm{RL}^{(1)}$. Más recientemente, se ha propuesto una nueva clasificación que, con el objetivo de clarificar el diagnóstico, agrupa todas las lesiones similares al LPO en una nueva llamada Enfermedad Liquenoide Oral (OLD) ${ }^{(5-6)}$.

Una de las complicaciones más importantes según el progreso y el pronóstico del LPO es la aparición de un carcinoma oral de células escamosas (COCE). En 1910 se documentó el primer caso de COCE sobre un LPO y desde entonces se han publicado numerosos estudios con el fin de esclarecer el índice de malignización del LPO, estableciéndose entre el 0 y el $5 \%$ y siendo motivo de controversia ${ }^{(7)}$. La predicción del potencial maligno del LPO es complicado debido a que sus características se solapan con las de las reacciones liquenoides y a la ausencia de unos criterios diagnósticos universales para su diagnóstico ${ }^{(2,4-7)}$.

El objetivo de esta revisión es evaluar la evidencia relacionada con la malignización del LPO, así como las características clínicas y la incidencia de las lesiones que pueden acabar desarrollando un COCE. Formulando la pregunta PICO: ¿Cuál es la incidencia de malignización del LPO y que factores asociados existen para la aparición de ésta?

\section{MATERIAL Y MÉTODO}

Se revisa la base de datos MEDLINE PubMed de los últimos 5 años (2013-2018) utilizando las si- 
guientes palabras clave: "malignant transformation of oral lichen planus" and "squamous cell oral carcinoma".

Los criterios de inclusión fueron: publicaciones en inglés o español, estudios humanos, metaanálisis, estudios de cohortes, revisiones sistemáticas y revisiones bibliográficas. Si bien comparamos únicamente los estudios clínicos.

Los criterios de exclusión fueron: estudios animales o "in vitro" que no se centren en el objetivo de esta revisión.

Se aplicó la declaración PRISMA en la presenta revisión (Preferred Reporting Items for Systematic reviews and Meta-Analyses) ${ }^{(8)}$.

\section{RESULTADOS}

Se obtuvieron 48 artículos de los cuales tras la lectura de resúmenes y la aplicación de nuestros criterios de inclusión y exclusión obtuvimos un total de 19 artículos. Además, añadimos 4 artículos mediante búsqueda manual. La revisión se basa en la comparación de 9 estudios de cohortes para analizar la incidencia de la progresión de LPO a COCE teniendo en cuenta las características clínicas, el sexo, el criterio diagnóstico utilizado y los factores de riesgo relacionados. (Figura 1)

En base a la bibliografía existen varios factores de riesgo relacionados con la capacidad de malignización del LPO como puede ser la DM, la hipertensión o los hábitos tóxicos entre otros (Tabla 2).

La Tabla $3^{(2,7,9-15)}$ muestra las características de los 9 estudios analizados comparativamente. Se estudiaron un total de 3.578 pacientes de los cuales 1.174 eran hombres, 2.001 mujeres y 303 no se especificaron. De este total de pacientes 3.430 fueron diagnosticados de LPO y 148 de RL. El rango de transformación maligna de LPO observado en los estudios varía entre 0.52 y $3.9 \%$. En esta revisión hemos encontrado que 54 de los 3578 pacientes desarrollaron un COCE en lesiones de LPO obteniendo un porcentaje de malignización del $1.5 \%$. Además, podemos observar que existen localizaciones con mayor predilección para la transformación maligna. Como muestra la Tabla 4, la lengua fue la localización con más episodios de maligni-

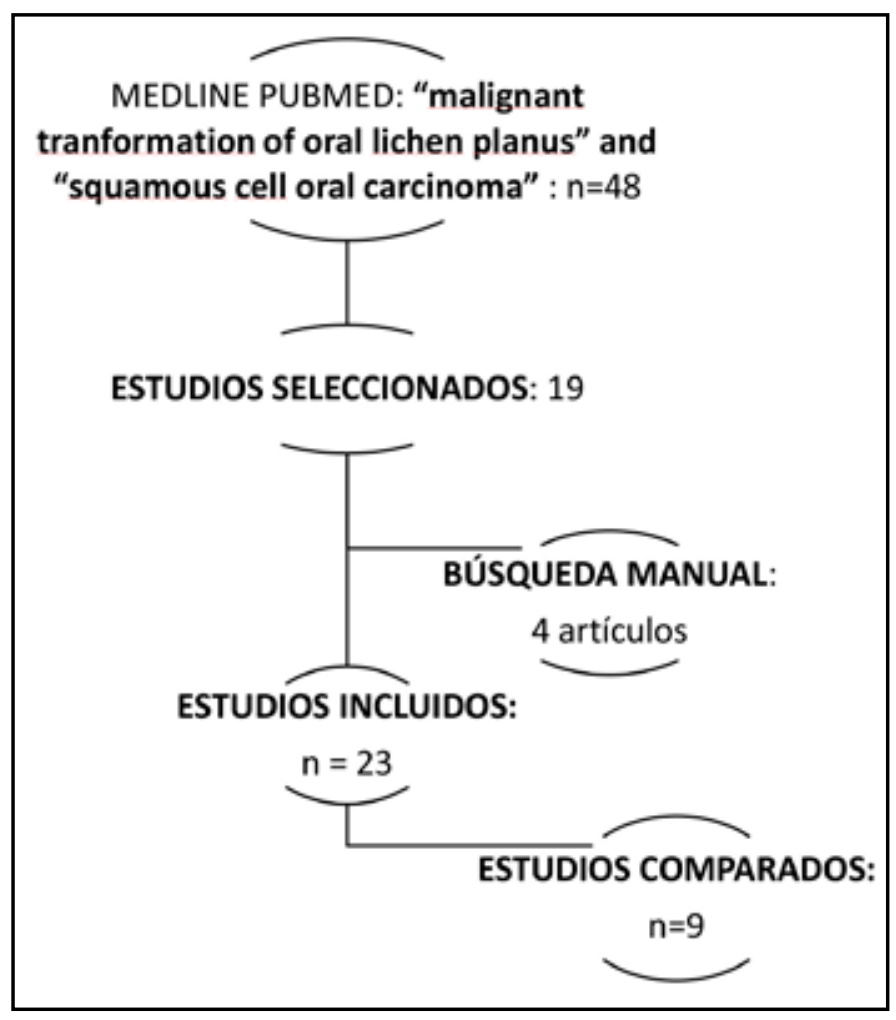

Figura 1. Diagrama de flujo de la búsqueda realizada.

\begin{tabular}{|l|l|}
\hline \multirow{2}{*}{$\begin{array}{l}\text { Criterios } \\
\text { clínicos }\end{array}$} & Presencia bilateral de lesiones simétricas \\
\cline { 2 - 2 } & $\begin{array}{l}\text { Presencia de lesiones reticulares blanquecinas pudiendo existir } \\
\text { lesiones erosivas, ampollas o en forma de placa concomitantes }\end{array}$ \\
\hline \multirow{2}{*}{$\begin{array}{l}\text { Criterios } \\
\text { histológicos }\end{array}$} & $\begin{array}{l}\text { Presencia de una banda definida de infiltrado inflamatorio } \\
\text { linfocitario en el tejido conectivo }\end{array}$ \\
\cline { 2 - 2 } & Signos de degeneración hidrópica de la capa basal \\
\cline { 2 - 2 } & Ausencia de signos de displasia epitelial \\
\hline
\end{tabular}

Tabla 1. Criterios diagnósticos del LPO y RL de van der Meij et al $(2003)^{3}$.

\begin{tabular}{|c|c|}
\hline \multicolumn{2}{|c|}{ FACTORES DE RIESGO $^{|2|}$} \\
\hline \multicolumn{2}{|c|}{ ALCOHOL $^{12,13,20,21}$} \\
\hline \multicolumn{2}{|c|}{ TABACO $^{12,13,20,21}$} \\
\hline \multirow{2}{*}{ INFECCIONES VÍRICAS $^{12,13,20,21}$} & VCH \\
\cline { 2 - 2 } ENFERMEDADES SISTÉMICAS & VPH \\
\hline \multirow{2}{*}{ CANDIDASIS $^{21}$} & DM \\
\cline { 2 - 2 } & HTA \\
\hline \multicolumn{2}{|c|}{ LOCALIZACIÓN $^{20,21}$} \\
\hline
\end{tabular}

Tabla 2. Factores de riesgo para la malignización del LPO.

zación seguida de la mucosa bucal y la encía. El suelo de la boca, que se considera como una localización crítica para las lesiones potencial- 


\begin{tabular}{|c|c|c|c|c|c|c|c|c|c|c|}
\hline \multirow{2}{*}{ Autor } & \multicolumn{2}{|c|}{$\mathrm{N}(\mathrm{LPO})$} & \multirow{2}{*}{$\begin{array}{l}\text { Tipo de } \\
\text { estudio }\end{array}$} & \multirow{2}{*}{$\begin{array}{c}\text { Criterios } \\
\text { diagnósticos }\end{array}$} & \multicolumn{2}{|c|}{ Sexo } & \multirow{2}{*}{$\begin{array}{c}\text { Casos de } \\
\text { transformación } \\
\text { maligna }\end{array}$} & \multirow{2}{*}{\multicolumn{2}{|c|}{$\begin{array}{c}\% \text { de } \\
\text { malignización }\end{array}$}} & \multirow[b]{2}{*}{ DM } \\
\hline & LPO & RL & & & $\mathrm{H}$ & M & & & & \\
\hline Lanioz et $\mathrm{al}^{9}$ & \multicolumn{2}{|c|}{303} & Cohortes & $\begin{array}{l}\text { WHO (revi- } \\
\text { sado) }\end{array}$ & & 7 & \multicolumn{2}{|c|}{2.35} & - \\
\hline $\begin{array}{c}\text { Gonzalez } \\
\text { Moles et al. }{ }^{10}\end{array}$ & 21(LPO) & $81(\mathrm{RL})$ & Cohortes & $\begin{array}{l}\text { Vander meij } \\
\text { et al. }\end{array}$ & 26 & 76 & 4 & \multicolumn{2}{|c|}{3.95} & - \\
\hline $\begin{array}{l}\text { Bombeccari } \\
\text { et al. } .^{2}\end{array}$ & 327 & - & Cohortes & $\begin{array}{c}\text { Vander meij } \\
\text { et al. }\end{array}$ & 98 & 229 & 8 & \multicolumn{2}{|c|}{$2.44 \%$} & 2 \\
\hline $\begin{array}{c}\text { Casparis et } \\
\text { al. }^{11}\end{array}$ & \multicolumn{2}{|c|}{542} & Cohortes & $\begin{array}{l}\text { Vander meij } \\
\text { et al. }\end{array}$ & 207 & 335 & $10(\mathrm{LPO})$ & $\begin{array}{l}4.4 \% \\
\mathrm{RL}\end{array}$ & $\begin{array}{l}1.25 \\
\text { LPO }\end{array}$ & 3 \\
\hline $\begin{array}{l}\text { A. Bermejo } \\
\text { Fenoll et al. }{ }^{12}\end{array}$ & \multicolumn{2}{|c|}{550} & Cohortes & WHO (1978) & 128 & 442 & 5 & \multicolumn{2}{|c|}{$0,90 \%$} & 4 \\
\hline $\begin{array}{l}\text { Van der Meij } \\
\text { et al. }{ }^{7}\end{array}$ & 125 & 67 & Cohortes & $\begin{array}{l}\text { WHO (revi- } \\
\text { sado) }\end{array}$ & 64 & 128 & $4(\mathrm{RL})$ & $2.1 \%$ & $\begin{array}{r}0 \% \\
\text { LPO } \\
\end{array}$ & \\
\hline $\begin{array}{l}\text { Zheng-yu } \\
\text { Shen et al. }{ }^{13}\end{array}$ & \multicolumn{2}{|c|}{518} & Cohortes & WHO (1978) & 353 & 165 & 5 & \multicolumn{2}{|c|}{$0.96 \%$} & 7 \\
\hline Wang et al. ${ }^{14}$ & \multicolumn{2}{|c|}{381} & Cohortes & $\cdot$ & 149 & 232 & 2 & \multicolumn{2}{|c|}{$0.52 \%$} & - \\
\hline $\begin{array}{l}\text { Buidmir } \\
\text { et al. }{ }^{.15}\end{array}$ & \multicolumn{2}{|c|}{663} & Cohortes & WHO (1978) & 149 & 414 & 4 & \multicolumn{2}{|c|}{$0.7 \%$} & - \\
\hline & \multicolumn{2}{|c|}{3578} & - & - & 1174 & 2001 & 54 & \multicolumn{2}{|c|}{$1.5 \%$} & 16 \\
\hline
\end{tabular}

Tabla 3. Parámetros analizados en los 9 artículos estudiados.

\begin{tabular}{|c|c|c|c|c|c|c|}
\hline Autor & $\begin{array}{l}\text { Suelo } \\
\text { de la } \\
\text { boca }\end{array}$ & Encía (N) & $\begin{array}{c}\text { Mucosa } \\
\text { bucal (N) }\end{array}$ & Lengua $(\mathrm{N})$ & Paladar (N) & Labio (N) \\
\hline Lanioz et $\mathrm{al}^{9}$ & - & 1 & 2 & 4 & 1 & - \\
\hline $\begin{array}{c}\text { González-Moles } \\
\text { et al. }{ }^{10}\end{array}$ & 2 & 2 & - & - & - & - \\
\hline Bombeccari et al. ${ }^{2}$ & - & - & 2 & 3 & - & - \\
\hline Casparis et al. ${ }^{11}$ & - & 2 & 1 & 1 & 1 & - \\
\hline $\begin{array}{c}\text { A. Bermejo-Fenoll } \\
\text { et al. }{ }^{12}\end{array}$ & - & - & 2 & 3 & - & - \\
\hline $\begin{array}{l}\text { Van der Meij } \\
\text { et al. }{ }^{17}\end{array}$ & - & 1 & 2 & 1 & - & 1 \\
\hline $\begin{array}{l}\text { Zheng-yu Shen } \\
\text { et al. }{ }^{13}\end{array}$ & - & - & - & 2 & - & - \\
\hline Whang et al. ${ }^{13}$ & - & - & - & 2 & - & - \\
\hline Buidmir et al. ${ }^{15}$ & - & - & - & - & - & - \\
\hline
\end{tabular}

Tabla 4. Localización de los COCE desarrollados en nuestra revisión.

mente malignas, tan solo está afectada en 2 casos según nuestra revisión. El labio y el paladar tampoco presentan gran afectación.

En los estudios en los que se estudiaron también los casos de RL y se analizó su rango de malignización ${ }^{(7,11)}$ observamos una mayor incidencia de malignización $\left(2.1 \%^{(7)}\right.$ y $\left.4.4 \%^{(11)}\right)$.

Respecto a los criterios diagnósticos utilizados en los estudios podemos observar que no existe un acuerdo universal puesto que no todos utilizan los mismos (Tabla 3). Asimismo, y aunque no todos los estudios especifican las patologías que presentan los pacientes que experimentan la malignización de sus lesiones, 16 de los 54 casos de malignización padecían DM.

\section{DISCUSIÓN}

El mayor riesgo que existe en los pacientes diagnosticados de LPO es la evolución maligna de sus lesiones ${ }^{(5)}$. La frecuencia estimada de este suceso varia del 1 al $5 \%$ de los casos ${ }^{(7)}$. Existe, como vemos, controversia debido a las variaciones existentes en las diferentes publicaciones ${ }^{(5)}$. Recientemente solo se han publicado dos revisiones sistemáticas ${ }^{(16,17)}$ que han obtenido un porcentaje de malignización del 1.09 y un $1.40 \%$ respectivamente, rango que en nuestra revisión de 9 artículos es del 1.5\% para pacientes diagnosticados de LPO que desarrollan un COCE, sin tener en cuenta los casos de RL que malignizaron. En este sentido Aghbari et al. (18) también encontraron un índice de malignización similar al nuestro (1.1\% para LPO).

La presentación demográfica más típica del LPO es el sexo femenino y la octava década de vida ${ }^{(16)}$. En nuestra revisión encontramos que el $55.9 \%$ de los casos de LPO se dio en mujeres y, como podemos observar en la Tabla 4, destaca la lengua como localización más frecuente de malignización (47.8\%) seguido de la mucosa bucal con un $25.6 \%$, la encía con un $17 \%$, suelo de la boca y paladar con un $5 \%$ y un $2.5 \%$ en los labios, al igual que Casparis et al. ${ }^{(11)}$. Por el contrario, Aghbari et al. ${ }^{(18)}$ no encontraron diferencias significativas en la prevalencia de malignización entre hombres y mujeres pese a ser más elevada en el sexo masculino.

Laniosz et al. ${ }^{(9)}$ observaron que la mayoría de 
las lesiones que presentaron malignización eran erosivas, datos semejantes a Bombeccari et al. (2) y Fitzpatrick et al. (16). Por otro lado, Van der Meij et al. ${ }^{(7)}$ hicieron un seguimiento de 5 años a un total de 192 pacientes y no encontraron ninguna malignización de lesiones de LPO; sí obtuvieron, en cambio, un $2.1 \%$ de casos de malignización de RL. De manera similar, Casparis et al. ${ }^{(9)}$, encontraron también diferencias entre RL y LPO, con porcentajes menores para liquen plano oral $(1.2 \%)$ que para las reacciones liquenoides $(4.4 \%)$. Cortés et al. ${ }^{(6)}$ concluyen en su revisión que hay una diferencia clara en el pronóstico de las lesiones y, coincidiendo con lo anteriormente expuesto, las RL presentan un mayor índice de malignizaciones que las lesiones de LPO y es por este motivo que se propone la nueva clasificación para las lesiones de LPO. (5-6) Así pues, observamos que uno de los mayores obstáculos existentes en la discusión del posible potencial maligno del LPO es debido a la ausencia de unos criterios diagnósticos claros. ${ }^{(5,6,10,11,16,19)}$ En nuestra revisión la mayoría de los estudios utilizaban los criterios diagnósticos propuestos por Van de Meij et al. ${ }^{(4)}$, pero dos de ellos ${ }^{(13,15)}$ utilizaron otros más antiguos propuestos por la WHO en $1978^{(3)}$. Por el contrario, Wang et al. ${ }^{(14)}$ no los especificaron. Esta heterogeneidad diagnóstica podría sesgar los estudios que estudian la malignización del LPO.

Se han asociado diversos factores de riesgo para la malignización del LPO (Tabla 2) siendo el tabaco y alcohol los más relacionados con la aparición del cáncer oral según la literatura ${ }^{(20-21)}$. Sin embargo, la mayoría de los pacientes diagnosticados de LPO y estudiados por Budimir et al. ${ }^{(15)}$ no eran fumadores, así como tampoco consumidores de alcohol. Laniosz et al. ${ }^{(9)}$ tampoco encontraron una relación directa entre el tabaco y el alcohol, resultados coincidentes a los obtenidos por Shen et al. ${ }^{(13)}$, A. Bermejo-Fenoll et al. ${ }^{(12)}$ y González-Moles et al. ${ }^{(10)}$. En controversia encontramos a Casparis et al. ${ }^{(11)}$, que en su estudio aceptaron que el hábito tabáquico se relaciona con una mayor severidad diagnóstica coincidiendo con la mayor evidencia consultada ${ }^{(18)}$. Los autores concluyen que, comparado con los no fumadores, los pacientes fumadores presentan un $\mathrm{OR}=2$ y los consumidores de alcohol un $\mathrm{OR}=3.52$.

Dentro de los factores de riesgo también se ha estudiado la presencia del virus la hepatitis C
(VHC) y la sobreinfección por Cándida de las lesiones ${ }^{(21)}$. Sobre la presencia del LPO y VHC Cassol et al. (22) encontraron una mayor prevalencia de VHC en pacientes afectados de LPO, pero en nuestro estudio tan solo dos ${ }^{(2,12)}$ tienen en cuenta la presencia de marcadores de VHC. Bermejo-Fenoll et al. ${ }^{(12)}$ obtuvieron resultados negativos para la infección de hepatitis $C$ en un $80.2 \%$, positivos en el $2.91 \%$ y no analizado en el resto en el 16.93\%. Ninguno de los pacientes afectados por VHC desarrollaron COCE; al igual que Bombeccari et al. ${ }^{(2)}$, que tampoco obtuvo ninguna malignización. Contrariamente, Aghbari et al. (18) sí que encuentra una asociación significativa entre la infección de VHC y la transformación maligna del LPO. La sobreinfección por Cándida puede promover la transformación neoplásica maligna debido a su capacidad de producir nitrosaminas e inflamación crónica una vez penetra en el epitelio ${ }^{(17)}$, pero ninguno de los estudios publicados recientemente correlaciona la candidiasis con la malignización del LPO.

Las características médicas de los pacientes también deben tenerse en cuenta en el estudio de los factores de riesgo. En este sentido, la diabetes mellitus (DM) juega un papel importante ya que se trata de una patología con gran incidencia en la población y se ha sugerido su posible relación con la malignización del LPO (13) aunque no existe evidencia significativa que lo pueda corroborar ${ }^{(20)}$. En nuestra revisión no hemos encontrado ningún estudio que relacione directamente los resultados obtenidos con la presencia de esta enfermedad, aunque dos de los casos de COCE obtenidos por Bombeccari et al. (2) presentaban DM, así como Casparis et al. ${ }^{(11)}$ y Bermejo-Fenoll et al. ${ }^{(12)}$ que obtuvieron 3 y 4 casos con DM respectivamente. Sin embargo, no encontramos una relación directa, así como tampoco una asociación clara entre la DM y la malignización del LPO, al igual que Shen et al. ${ }^{(13)}$, Aghbari et al. ${ }^{(18)}$ que tampoco relacionan la $\mathrm{DM}$ en su metaanlálisis. Otero et al. ${ }^{(23)}$, realizaron muy recientemente un metaanálisis en relación con la DM y la malignización del LPO obtuvieniendo resultados contradictorios, lo que nos indica que son necesarios más estudios con criterios diagnósticos similares.

Pese a que el hipertiroidismo y la artritis también han sido relacionadas con el LPO y su malignización, ninguno de los artículos estudiados apor- 
ta resultados que lo relacionen. La hipertensión también parece jugar un papel en el campo de la malignización del LPO ${ }^{(2)}$. Shen et al. ${ }^{(13)}$ concluye que la hipertensión podría no jugar ningún rol en la patogenia del LPO.

En el campo de las lesiones precancerosas se ha postulado que las terapias inmunosupresoras pueden incrementar el riesgo de cáncer oral en pacientes con LPO. Solo Bombeccari et al. ${ }^{(2)}$ estudiaron esta característica sin encontrar relación alguna al comparar pacientes tratados mediante terapia inmunosupresora y los no tratados.

Por otro lado, también se discute el papel de la terapia antiinflamatoria crónica aplicada en los episodios sintomatológicos del LPO. Todos los pacientes que presentaron malignización en el estudio de González-Moles et al. ${ }^{(10)}$ y Van der Meij et al. (4) habían recibido tratamiento mediante corticoides por lo que nuestros resultados sugieren que la aplicación de corticoides de manera tópica no tiene influencia en el riesgo de cáncer oral e incluso algunos autores mencionan la posibilidad de que el manejo crónico a bajas dosis con los mismos pudiese ser protector.

\section{CONCLUSIONES}

Nuestra revisión sustenta la teoría de que una proporción de lesiones diagnosticadas de LPO $(1.5 \%)$ sufren una transformación maligna desarrollando carcinoma oral de células escamosas (COCE). Esta malignización presenta una mayor incidencia en pacientes del sexo femenino. También se presenta una relación con el hábito tabáquico y alcohólico que según los datos obtenidos no podemos confirmar, así como tampoco la hipotética relación entre la infección por Cándida o VHC con una mayor malignización del LPO.

Son necesarios estudios basados en criterios diagnósticos coincidentes que investiguen la incidencia de la transformación maligna, así como el efecto del tabaco, el alcohol y las infecciones $y$ el resto de los factores de riesgo en el potencial maligno del LPO.

\section{BIBLIOGRAFIA}

1. Olson MA, Rogers RS, Bruce AJ. Oral lichen planus. Clin Dermatol 2016; 34(4):495-504.

2. Bombeccari GP, Guzzi G, Tettamanti M, Giann AB, Baj A, Pallotti F, et al. Oral lichen planus and malignant transformation: A longitudinal cohort study. Oral Surgery Oral Med Oral Pathol Oral Radiol Endod 2011; 112(3): 328-34.

3. Alrashdan MS, Cirillo N, McCullough M. Oral lichen planus: a literature review and update. Arch Dermatol Res 2016; 308(8): 539-51.

4. Van der Meij EH, Van der Waal I. Lack of clinicopathologic correlation in the diagnosis of oral lichen planus based on the presently available diagnostic criteria and suggestions for modifications. J Oral Pathol Med 2003; 32(9): 507-12.

5. Aguirre Urizar JM. Letter to the editor: oral lichenoid disease. A new classification proposal. Med Oral Patol Oral Cir Bucal 2008; 13(4): 224.

6. Cortés-Ramírez DA, Gainza-Cirauqui ML, Echebarria-Goikouria MA, Aguirre-Urizar JM. Oral lichenoid disease as a premalignant condition: the controversies and the unknown. Med Oral Patol Oral Cir Bucal 2009; 14(3):118-22.

7. Van der Meij EH, Mast H, Van der Waal I. The possible premalignant character of oral lichen planus and oral lichenoid lesions: A prospective five-year follow-up study of 192 patients. Oral Oncol 2007; 43(8): 742-8.

8. Urrutia G, Bonfill X. Declaración PRISMA: una propuesta para mejorar la publicación de revisiones sistemáticas y metaanálisis. Med Clin (barc) 2010; 135(11): 507-11.

9. Laniosz V, Torgerson RR, Ramos-Rodriguez AJ, Ma JE, Mara KC, Weaver AL, et al. Incidence of squamous cell carcinoma in oral lichen planus: a 25-year population-based study. Int J Dermatol 2019; 58(3): 296-301.

10. Gonzalez-Moles MA, Gil-Montoya JA, Ruiz-Avila I, Bravo M. Is oral cancer incidence among patients with oral lichen planus/ 
oral lichenoid lesions underestimated? J Oral Pathol Med 2017; 46(2): 148-53.

11. Casparis S, Borm JM, Tektas S, Kamarachev J, Locher MC, Damarau G, et al. Oral lichen planus (OLP), oral lichenoid lesions (OLL), oral dysplasia, and oral cancer: retrospective analysis of clinicopathological data from 2002-2011. Oral Maxillofac Surg 2015; $19(2) ; 149-56$.

12. Bermejo-Fenoll A, Sanchez-Siles $M$, López-Jornet P, Camacho-Alonso F, Salazar-Sanchez N. Premalignant nature of oral lichen planus. A retrospective study of 550 oral lichen planus patients from south-eastern Spain. Oral Oncol 2009; 45(8): 54-6.

13. Shen ZY, Liu W, Zhu LK, Feng JQ, Tang GY, Zhou ZT. A retrospective clinicopathological study on oral lichen planus and malignant transformation: Analysis of 518 cases. Med Oral Patol Oral Cir Bucal 2012; 17(6): 943-7.

14. Wang YY, Tail YH, Wang WX, Chen CY, Kao $\mathrm{YH}$, Chen YK, et al. Malignant transformation in 5071 southern Taiwanese patients with potentially malignant oral mucosal disorders. BMC Oral Health 2014; 14(1):1-9.

15. Budimir V, Richter I, Andabak-Rogulj A, Vucicevic-Boras V, Budimir J, Brailo V. Oral lichen planus - Retrospective study of 563 Croatian patients. Med Oral Patol Oral Cir Bucal 2014; 19(3): 1-6.

16. Fitzpatrick SG, Hirsch SA, Gordon SC. The malignant transformation of oral lichen planus and oral lichenoid lesions. A systematic review. J Am Dent Assoc 2014; 145(1): 45-56.

17. Giuliani M, Troiano G, Cordaro M, Corsalini M, Gioco G, Lo Muzio L, et al. Rate of malignant transformation of Oral Lichen Planus: a systematic review. Oral Dis 2019; 25(3): 693-709.
18. Aghbari SMH, Abushouk Al, Attia A, Elmaraezy A, Menshawy A, Ahmed MS, et al. Malignant transformation of oral lichen planus and oral lichenoid lesions: A meta-Analysis of 20095 patient data. Oral Oncol 2017; 68 (May): 92-102.

19. Van Der Waal I. Oral potentially malignant disorders: Is malignant transformation predictable and preventable? Med Oral Patol Oral Cir Bucal 2014; 19(4): 1-5.

20. Porter S, Gueiros LA, Leão JC, Fedele S. Risk factors and enteropathogenesis of potentially premalignant oral epithelial lesions. Oral Surg Oral Med Oral Pathol Oral Radiol 2018;125(6): 603-11.

21. Agha-Hosseini F, Sheykhbahaei N, SadrZadeh-Afshar MS. Evaluation of Potential Risk Factors that contribute to Malignant Transformation of Oral Lichen Planus: A Literature Review. J Contem Dent Pract 2016; 17(8): 692-701.

22. Cassol J, Rodríguez-de Rivera ME, Otero EM, Estrugo A, Jané E, López J. Oral lichen planus and its relationship with systemic diseases. A review of evidence. J Clin Exp Dent 2018; 10(9): 938-44.

23. Otero Rey EM, Yáñez-Busto A, Rosa Henriques IF, López-López J, Blanco-Carrión A. Lichen planus and diabetes mellitus: Systematic review and meta-analysis. Oral Dis 2018; (Sep 11).

\section{AUTOR DE CORRESPONDENCIA:}

José López López

Facultad de Medicina y Ciencias de la Salud (Odontología), Universidad de Barcelona

Campus Universitario de Bellvitge

Pabellón de Gobierno

C/ Feixa LLarga s/n 08907

L'Hospitalet de Llobregat Barcelona - España

18575jll@gmail.com 